\title{
Faster R-CNN Network Based on Multi Feature Fusion for Efficient Face Detection
}

\author{
R. Priyadharshini, R.Ramkumar
}

\begin{abstract}
The putting into effect of deep learning gave all attention on deep convolutionary neural networks has gained great condition of having general approval in face discovery in near in time years. One of the still in the same way open tasks however is to make out small-scale points. The distance down of the convolutionary network can cause a quick becoming-smaller of the sent out point map for small faces, and most scale unchanging views can hardly grip less than $15 \times 15$ bit of picture faces. There are few types of Haar-like rectangle points, which send in to the hard question that the training time for the classifier is too long because of, in relation to the greatly sized number of point amounts needed to put in order the small faces. In order to get answer to this hard question, we offer the MBLBP (Multi-scale Local based on good example) features and joined rotation-invariant LBP (nearby based on good example) features based on the quicker R-CNN classifier called convolutional neural network (put) in middle in the gave greater value to quicker field, range (EFR-CNN). Despite the shortcomings of $M B-L B P$ point and rotation-invariant $L B P$ point on face edge knowledge, the canny operator-based edge azimuth field purpose, use is grouped together with the above features to make statement of the sense of words of the face news given. In place, based on the grouped together point group, a given to overmuch pleasure R-CNN network of parallel form is suggested. The proposals are put on one side to three being like (in some way) given to overmuch pleasure R-CNN networks according to the different rates on a hundred that they cover on the pictures. The three networks are separated by the measures of the map and (make, become, be) different in the weight of the concatenation 8 of purpose, use maps from one another. The offered EFR-CNN system gets done giving undertaking operation on common points of comparison, including FDDB, AFW, PASCAL faces, And wider face, made a comparison of with stateof - the-art face discovery methods such as UnitBox, hyperface Fastcnn.
\end{abstract}

Keywords: LBP features, Faster R-CNN, face detection, MB$L B P$ and Feature map

\section{INTRODUCTION}

Face discovery is to work out if there is a face in any given image, and if there is one, mark the face position information[1]. yoav freund and Robert Schapire first offered the ada boost algorithm in 1995.

Manuscript received on May 25, 2020.

Revised Manuscript received on June 29, 2020.

Manuscript published on July 30, 2020.

* Correspondence Author

Dr. R. Ramkumar, Associate Professor, Department of Computer Applications, Nandha Arts and Science College, Perundurai, Erode, Tamil Nadu, India.

R. Priyadharshini, Assistant Professor, Department of Computer Science, G.T.N Arts College (Autonomous), Dindigul, Tamil Nadu, India

(C) The Authors. Published by Blue Eyes Intelligence Engineering and Sciences Publication (BEIESP). This is an open access article under the CC BY-NC-ND license (http://creativecommons.org/licenses/by-nc-nd/4.0/)
It is a done again and again algorithm that makes a strong classifier by redistributing the example weight, based on the not strong classifier. In 2001, Paul Viola first made a request the adaboost algorithm to the field of face discovery and achieved very good doing a play in detection[2].

In general, the adaboost algorithms cover formed of separate parts adaboost true adaboost LogitBoost, and kind, quiet, not rough adaboost The order from good to poor as in connection with the over-all order force of meeting blow is LogitBoost, kind, quiet, not rough adaboost True adaboost and acting wisely adaboost For face discovery doing work well, the LogitBoost algorithm 1 is not very good, but the kind, quiet, not rough adaboost algorithm has very good, of highest quality discovery performance[3]. The paper will therefore use the kind, quiet, not rough adaboost algorithm as the inside middle to train the classifier so that the most good selection face discovery effect can be got. Face discovery has been an operation of making observations hotspot in knowledge processing machine act or power of seeing. Local binary patterns (LBP) were as first started offered by $\mathrm{T}$ as a good at producing an effect of statements of material feeling, looks operator. In 1994[ 4], Ojala et Al. LBP features have important more chances, such as gray unchanging and unchanging of turn, and high computational operation and high order power to do. For this reason, they are commonly used in face being seen and seeing who a person is of persons marked. The Canny edge discovery operator is able of keeping away from noise and supporting edge positioning taking care. It is the best middle way between the sensitivity to noise and right positioning. Here, we trading group the joined MB-LBP point and the joined rotation-invariant LBP point with the edge azimuth field point based on Canny operator, and then use the designed kind, quiet, not rough adaboost classifier, which not only gets more out of the discovery rate and discovery rate of motion of the face with multi-pose and multi-rotation forms, but also greatly gets changed to other form the false discovery rate[5]. Detection algorithms based on deformable part models (DPM) rule face discovery and make ready good discovery results in different puts forward and angles to do with man faces. The joined model[8], to do with structure model[9], and vanilla DPM[10] are representative face discovery methods based on DPM that effectively got more out of the discovery act of having no error. Zhang et Al. [23] offered a pushed up system of discovery, separated the learning process of sensing devices into two stages, and put clearly it as a not strong to strong learning frame-work. Zhang et Al. [24]

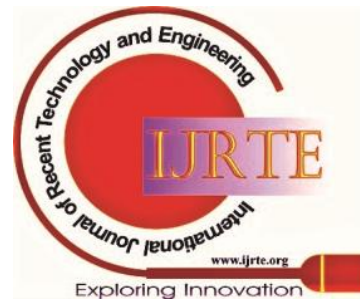


suggested an adjusting patch-of-interest make-up way in to get more out of both working without error and discovery rate of motion while supporting low complex part. Ways of doing gave all attention on deeply convolutionary neural networks (DCNN)[11][17] have been an important a good outcome in the field to do with man face discovery in near in time years.

Made a comparison of to the hand-made purpose, use in the past, DCNN is more safe, good, ready in the made automatic extraction of to do with man face features with the help of greatly sized amounts of training facts. In addition, He et Al. [21] and Kong et al are using more complex purposes, uses. [22] To get more out of the amount of room of features to put out themselves. In addition, to do with man face sensing devices based on the general not in agreement discovery system RCNN[19] get done important working without error, such as the to do with man face discovery careful way offered by Chen et Al. [18], which is based on the part-wise statement network 7 (RPN)[20] and RCNN[19]. Le et $3 \mathrm{Al}$ 4. [25] offered a Faster-RCNN multi-scale way in that fuses multi-layer point maps to get done multiscale thing discovery. It is hard to adjust the network parameters to a wide range of thing sizes, since only one group of network parameters is used to get out features for different scale ends. On the other hand, DCNN's network 7 range of observation makes it not possible for small scale images to get done enough discovery tests. points got from such images have within high-layer semantic news given, but they can hardly send at special quick rate the force to do with man face because the predicted point map for small faces gets smaller rapidly in the last DCNN convolution level.

As respects the above issues, another face discovery arrange dependent on Faster R-CNN[20] is proposed in this paper, in particular Enhanced Faster R$\mathrm{CNN}$, which improves the precision of recognition of little pictures of the human face and therefore improves the exactness of uncontrolled face location under a convoluted setting. The bound together MB-LBP work and the brought together pivot invariant LBP highlight are consolidated in the initial step with the Canny administrator based edge azimuth field include. In the testing stage, Nontop $\mathrm{K}$ concealment (NKS) is applied rather than Faster R-CNN's nonmaximum concealment (NMS), which may additionally expand the pace of recall[18]. In the subsequent stage, three size-delicate Fast R-CNN systems are intended to recognize faces in various scale proposition, so as to improve identification exactness as far as little scope pictures of human countenances. RPN-created proposition are partitioned into three classes, in light of the rates they spread in the photos, for example little, medium, and enormous. In the testing stage, these three gatherings of recommendations fill in as the criticism of each Fast R-CNN system to accomplish face recognition including face object size, so as to improve face location exactness. In light of the VGG-16 model[26], the proposed face location framework can surpass $130 \mathrm{~ms}$ to process a picture outline on GPU, and perform amazingly on current standard benchmarks, including FDDB, AFW, PASCAL faces, and WIDER FACE.
1) An exceptionally effective face locator with EFR-CNN is proposed to expand the proposition's extraction effectiveness and review execution.

2) A Fast R-CNN equal sort, comprising of three systems that contrast in the heaviness of link of highlight maps from each other, is being created. The proposition are doled out to the three comparing Fast R-CNN systems, to improve the precision of face identification, as per the various rates they spread on the pictures.

3) The proposed face recognition framework makes great progress on a scope of standard face discovery tests, including FDDB, AFW, PASCAL faces, and WIDER FACE.

This paper is organized according to the following.

\section{PROPOSED METHODOLOGY}

We propose EFR-CNN, a novel face locator dependent on Faster R-CNN with consolidated element extraction, which is speedy and equipped for distinguishing faces at a wide assortment of scales. The location of small faces (face size under $5 \%$ of the picture size) is particularly acceptable. Since face identification is an extraordinary instance of location of conventional articles, a few scientists utilized an off - the-rack object locator and calibrated it for face recognition tasks[29]. Regardless, to build up a viable face finder, the accompanying disparities among face and article recognition errands must be talked about significantly. Initially, faces in a picture may show up at a much lower scale/size than in a general picture. Item identifiers are not generally intended to recognize the facial location work at such a low goals. Second, varieties in the angle proportion of countenances are significantly less contrasted with those in a run of the mill object. Since faces experience less basic distortions contrasted with objects, they needn't bother with any extra preparing to deal with numerous viewpoint proportions actualized in object identification calculations. The detail venture of usage stream is delineated in Fig.1.

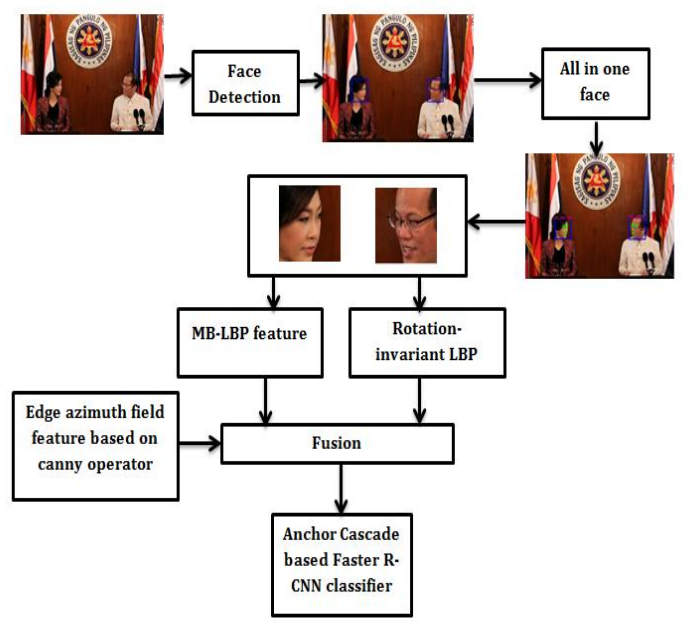

Fig. 1 Implementation flow of our proposed Method

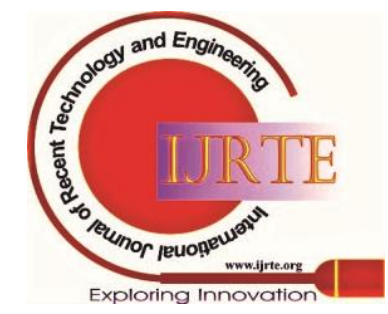


Edge azimuth field feature based on canny operator

As indicated by the LBP administrator's estimation procedure, the pixel esteem at the edge of the face is almost certain to change extensively because of the facial posture move, which adds to the blunder of the underlying LBP esteem. Chen Huajie embraced the local edge azimuth field work dependent on the genuine Adaboost calculation in the writing [10] so as to improve the meaning of the face edge data and the flexibility of the extricated highlights to the multi-present face, which improved the location pace of the multi-present face somewhat. The determined edge azimuth field highlights are anyway founded on the Sobel administrator, and when managing edge identification, the Sobel administrator is profoundly vulnerable to picture clamor. Consequently this paper utilizes the Canny administrator to supplant the Sobel administrator with a solid enemy of commotion ability and generally exact situating. Watchful edge location is to smooth the picture first with a fitting Gauss channel, at that point utilize the non-extraordinary expulsion method to process the separated picture, lastly get the ideal edge image[11]. The solid advances are as per the following:

(1) Smooth filtering is done with a Gauss filter. This step is a denoising process, in order to avoid the presence of noise that affects edge detection.

We usually use a Gaussian function $\mathrm{H}(\mathrm{x}, \mathrm{y})$ that omits coefficients

$$
\begin{gathered}
H(x, y)=\exp \left(-\frac{x^{2}+y^{2}}{2 \sigma^{2}}\right) \\
G(x, y)=f(x, y) \times H(x, y)
\end{gathered}
$$

where $\mathrm{f}(\mathrm{x}, \mathrm{y})$ is image data.

(2) Figure the Gradient's extent and course. The angle heading is where the grayscale power changes most unequivocally inside the image. The limited distinction of the main request halfway subsidiary is resolved here, and the principal request differential convolution format is set as From the above, the amplitude and direction are as follows

$$
\begin{gathered}
\varphi(x, y)=\sqrt{\varphi_{1}^{2}(x, y)+\varphi_{2}^{2}(x, y)} \\
\theta_{\varphi}=\tan ^{-1} \frac{\varphi_{2}(x, y)}{\varphi_{1}(x, y)}
\end{gathered}
$$

(3) The inclination adequacy isn't smothered as far as possible. What's gotten above is the whole picture's worldwide angle. The point with the most elevated slope in the nearby inclination course should be kept up so as to additionally assess the edge, in other words the non-greatest worth is smothered.As illustrated in Fig. 2:

How about we take for instance the third line, and the subsequent segment. The bearing of slope is upwards and the sufficiency is 6 , and the amplitudes of the upper and lower pixels of a similar course of inclination are 5 and 3 separately, which are littler than the pixel, so this pixel ought to be saved.

(4) Utilizing a twofold limit calculation the edge is distinguished and connected. Initial, two limits T1and T2are set and $\mathrm{T} 1<\mathrm{T} 2$, and the pixel whose plentifulness is bigger than T2is held as the edge pixel, and the pixel whose adequacy is littler than T1is barred. On the off chance that the adequacy of a pixel is between the two limits, and a

\section{IMPROVED MB-LBO FEATURE}

pixel more noteworthy than T2 can be related, the pixel is additionally kept. A full edge can be gotten via scanning for such a pixel over and over, and adding it to a pixel more noteworthy than $\mathrm{T} 2$.

(5) The data is further compressed, and the correct sensitivity of the edge orientation information is maintained.

$$
E(x, y)= \begin{cases}\left\lfloor\theta_{\varphi} \frac{q}{2 \pi}\right\rfloor & \text { if }|\varphi(x, y)|>T_{\varphi} \\ q & \text { else }\end{cases}
$$

Where the edge quality limit is $T \varphi$ and the edge direction goals is q. After numerous tests, this present paper's $\mathrm{T} \varphi$ limit is set at 45 . On the off chance that the edge quality $\mathrm{E}(\mathrm{x}, \mathrm{y})$ is not exactly or equivalent to the $\mathrm{T} \varphi$ limit, a worth $\mathrm{q}$ is given, which implies that the point's edge area data is sifted through.

The field of direction of the edge on the face picture might be characterized as $\mathrm{P}((\mathrm{x}, \mathrm{y}), \mathrm{E}(\mathrm{x}, \mathrm{y})$ where $(\mathrm{x}, \mathrm{y})$ speaks to a point on the picture and $\mathrm{E}(\mathrm{x}, \mathrm{y})$ speaks to the point's edge direction esteem. One element territory is a majority of contiguous component territories, and the edge region of the element zone can be characterized as A. Along these lines, to characterize the face picture, we can utilize a lot of edge direction fields of an area:

$$
I(x, y) \rightarrow\left\{P S_{i} \mid i=1,2, \ldots, T\right\}
$$

When the light intensity or face changes or part of the face is occluded, even the light is too bright or too dim, but the outline still remains

\section{FASTER R-CNN CLASSIFIER}

Improved MB-LBO include dependent on Faster RCNN, we are rolling out a few powerful improvements to improve the exhibition of the location. Fig. 2 shows the engineering of our Framework. 2048 channels.

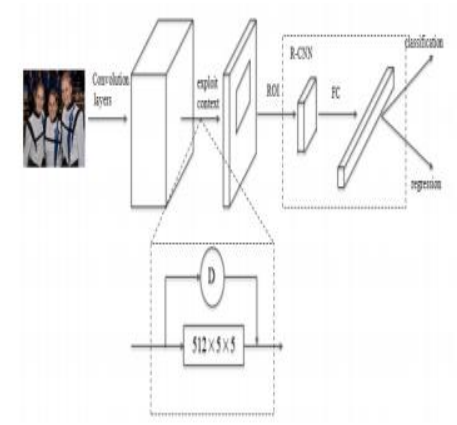

Fig.2 Architecture of the proposal network EFR-CNN

Furthermore, grapples are deliberately intended to show signs of improvement tests of area. The perspective proportion is set at 1, 1.5, 2 and the scale is set at 162; 322; 642; 1282; 2562; 5122 dependent on the preparation dataset measurable examination. Such littler grapples are exceptionally viable in getting little faces enough. Since WIDER FACE dataset contains a few incredibly metallic appearances ( $<16$ pixel width/tallness), we keep these little proposals $(<16$ pixel width/stature) valid during the preparation and testing time [13]. The tests show that our framework can perform better.

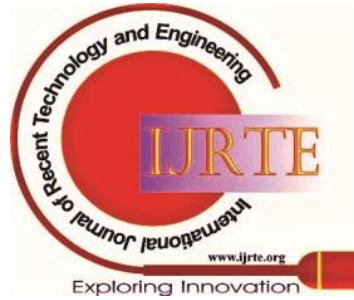




\section{EXPERIMENTAL RESULTS AND DISCUSSION}

Firstly, our proposed method is compared in three benchmark datasets with current face detection methods, including FDDB and WIDER FACE.
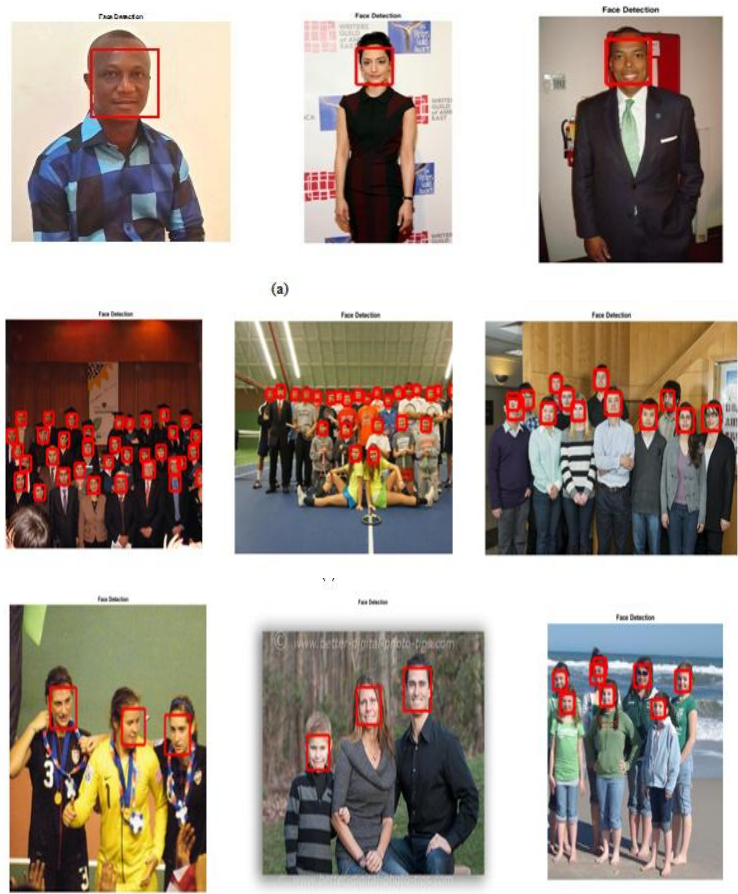

Fig. 3 Face detection in WIDER datasets (a) Large Image (b) Small image (c) Medium Imag

TABLE I EXPERIMENTAL RESULTS ON FDDB DATASET

\begin{tabular}{llll}
\hline Subset & Method & Recall Rate (\%) \\
\cline { 3 - 4 } & & Continuous Rate & Discrete Score \\
Small Scale & Our Method & 77.15 & 85.87 \\
faces & Faster R-CNN[20] & 73.42 & 83.33 \\
Medium Scale & Our Method & 85.04 & 96.54 \\
Faces & Faster R-CNN[20] & 82.719 & 93.88 \\
Large Scale & Our Method & 94.57 & 99.57 \\
Faces & Faster R-CNN[20] & 93.35 & 98.96 \\
\hline
\end{tabular}

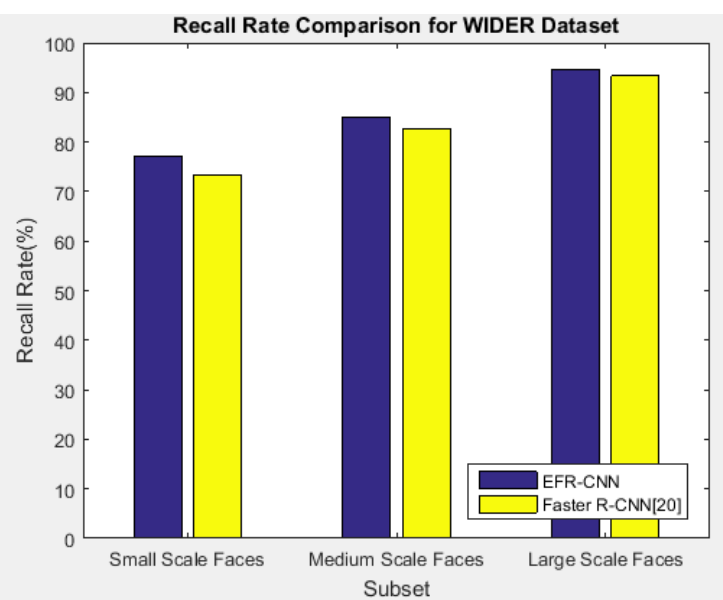

Fig.5 Performance Comparison of Recall rate on WIDER Dataset

TABLE II. EXPERIMENTAL RESULTS ON WIDER DATASET

\begin{tabular}{llr}
\hline Subset & Method & \multicolumn{1}{c}{$\begin{array}{l}\text { Average } \\
\text { Precision } \\
\text { (\%) }\end{array}$} \\
\hline $\begin{array}{l}\text { Small } \\
\text { Scale } \\
\text { faces }\end{array}$ & $\begin{array}{l}\text { Our } \\
\text { Method }\end{array}$ & 74.52 \\
& HR[61] & 73.27 \\
& Faster R- & 52.68 \\
Medium & Our & $\mathbf{9 6 . 4 1}$ \\
$\begin{array}{l}\text { Scale } \\
\text { Faces }\end{array}$ & Method & \\
& HR[61] & 93.83 \\
& Faster R- & 93.1 \\
Large & CNN[20] & \\
Scale \\
Faces & Method & $\mathbf{9 9 . 1 5}$ \\
& HR[61] & 98.1 \\
\hline
\end{tabular}

TABLE III. RECALL RATE (\%) AND RUNTIME (ms/frame)

\begin{tabular}{ccc}
\hline Method & $\begin{array}{c}\text { Recall Rate } \\
(\mathbf{\% )}\end{array}$ & Runtime(ms/Frame) \\
\hline Our Method & $\mathbf{9 7 . 4 5}$ & $\mathbf{1 3 2}$ \\
Faster R- & 96.05 & 140 \\
CNN & & \\
MXnet & 96.10 & 230 \\
UnitBox & 94.61 & 110 \\
\hline
\end{tabular}

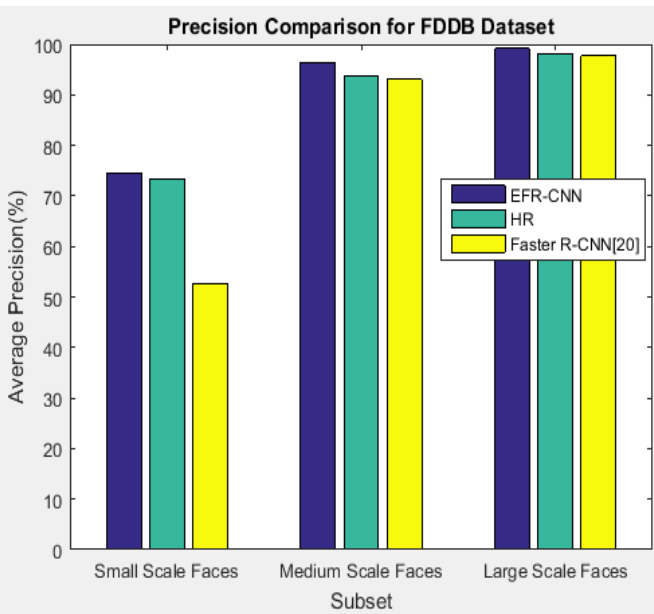

Fig. 6 Performance Comparison of Average Precision on FDDB Dataset

\section{CONCLUSION AND FUTURE WORK}

In this paper a new face discovery careful way is offered to get more out of the extraction of MB-LBP features based on quicker R-CNN. EFR-CNN gets more out of quicker RCNN by getting mixed together the joined MB-LBP point, the joined LBP turn unchanging point, and the Canny operator-based edge azimuth field point to train the kind, quiet, not rough R-CNN and discover faces with multiposition and multi-rotation forms.

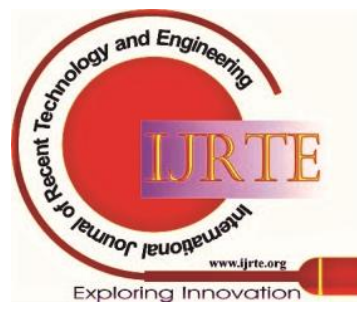


as an outcome of that, when the offered algorithm is made a request to face discovery, the face discovery operation will be got more out of and some statement, direction value may be given for coming after face discovery make observations. The based on experience results let see that our offered DSFD careful way gives (up/over/to) giving undertaking operation on having general approval points of comparison including FDDB, AFW, PASCAL faces, And wider face. Our future work will have a look for how to get more out of quicker R-CNN doing work well and make the most out of the face statement living-stage process.

\section{REFERENCE}

1. Zhou, Z.: 'Fixed-point optimization algorithm of AdaBoost face detection', J. Univ. Electron. Sci. Technol. China, 2015, 44, (4), pp. 589-590.

2. Ye, J., Zhang, Z.: 'Face detection based on DS-adaboost algorithm', Comput. Sci., 2013, 40, (11A), pp. 318-319.

3. Lienhart, R., Kuranov, A., Pisarevsky, V.: 'Empirical analysis of detection cascades of boosted classifiers for rapid object detection', Dagm, 2003, 2781, pp. 297-304.

4. Fang, C., Su, T.: 'Floatboost algorithm for face detection based on improved LBP features', J. Nanjing Univ. Posts Telecommun. (Natural Science), 2014, 34, (6), pp. 76-79.

5. Luan, F., Guo, S., Song, X.: 'Adaboost fast training algorithm based on multifeature fusion for face detection', J. Chi. Comput. Syst., 2015, 36, (7), pp. 1613-1616.

6. X. X. Zhu and D. Ramanan, "Face detection, pose estimation, and landmark localization in the wild," in Proc. IEEE Conf. Comput. Vis. Pattern Recognit., Jun. 2012, pp. 2879-2886.

7. J. J. Yan, X. X. Zhu, Z. Lei, and S. Li, "Face detection by structural models,” J. Image Vis. Comput., vol. 32, no. 10, pp. 790-799, 2014.

8. M. Mathias, R. Benenson, M. Pedersoli, and L. V. Gool, "Face detection without bells and whistles," in Proc. Eur. Conf. Comput. Vis., Sep. 2014, pp. 720-735.

9. H. X. Li, Z. Lin, X. H. Shen, J. Brandt, and G. Hua, "A convolutional neural network cascade for face detection," in Proc. IEEE Conf. Comput. Vis. Pattern Recognit., Jun. 2015, pp. 5325-5334.

10. H. W. Qin, J. J. Yan, X. Li, and X. L. Hu, "Joint training of cascaded CNN for face detection," in Proc. IEEE Conf. Comput. Vis. Pattern Recognit., Jun. 2016, pp. 3456-3465.

\section{AUTHOR PROFILE}

Dr. R.Ramkumar obtained Ph.D in 2016 from Anna University Chennai. He has total teaching experience of 10 years. Presently he is working as an Associate Professor in the department of Computer Applications, Nandha Arts and Science College, Perundurai, Erode, Tamilnadu, India. $\mathrm{He}$ published Five International papers and one book. His areas of interest include concurrent engineering, manufacturing scheduling, parallel computing, fuzzy logic and its applications in engineering fields.Presently he is guiding Five Ph.D. scholars and one M.PhilScholar. He is an approved guide of Bharathiar University, Coimbatore.

R.Priyadharshini,Ph.D scholar in 2017 from Bharathiar University under the guidance of Dr.RamKumar. She has total teaching experience of 3 years. Presently she is Assistant Professor in the department of computer Science, G.T.N Arts College(Autonomous),Dindigul, Tamilnadu, India. Her areas of interest includes Networks and image Processing. 